\title{
ON GEÖCZE'S PROBLEM FOR NON-PARAMETRIC SURFACES
}

\author{
BY \\ H. P. MULHOLLAND
}

1. Introduction. Suppose that $P$ stands for a (closed) polygonal region in the $(x, y)$-plane, and that we consider the portion

$$
S(f, P): \quad z=f(x, y) \quad[(x, y) \in P]
$$

of the surface $z=f(x, y)$, where

(1.2) $f(x, y)$ is everywhere a continuous and one-valued function of $(x, y)$. that

Now let $\left\{S\left(f_{n}, P\right)\right\}$ be a sequence of polyhedra inscribed to $S(f, P)$ such

(1.3) $f_{n}(x, y)$ is a linear function of $x$ and $y$ on every triangle in some triangulation $\left({ }^{1}\right) \mathcal{T}_{n}(P)$ of $P$, and $f_{n}(x, y)=f(x, y)$ at every vertex of $\mathcal{T}_{n}(P)$;

(1.4) the greatest diameter of any triangle of $\mathcal{T}_{n}(P)$ tends to 0 as $n \rightarrow \infty$.

Denoting by $E\left(f_{n}, P\right)$ the area of $S\left(f_{n}, P\right)$ (in the elementary sense), we define

$$
A^{*}(f, P)=\inf \lim \inf E\left(f_{n}, P\right) \quad(n \rightarrow \infty),
$$

the infimum being for all sequences $\left\{f_{n}(x, y)\right\}$ satisfying (1.3) and (1.4); and we denote by $A(f, P)$ the Lebesgue area of $S(f, P)$. The following theorem, settling Geöcze's problem $\left({ }^{2}\right)$ for non-parametric surfaces, has recently been established $\left({ }^{3}\right)$ : the object of the present paper is to give a new and comparatively short proof of it by following up an approach devised by Radó (cf. $[8$, V. 3.50-53, pp. 545-549]) in earlier work on this problem. The reader interested in the historical development of some of the ideas involved in this approach may read the papers by L. C. Young [11], H. D. Huskey [2], and T. Radó [7] listed at the end.

Presented to the Society, October 29, 1949; received by the editors September 20, 1949.

(1) By a triangulation of $P$ we shall understand a subdivision of $P$ into a finite number of nonoverlapping triangles such that no vertex of a triangle is an internal point of a side of another.

(2) An account of this problem and a restricted solution of it are given by T. Rado in Length and area $[8$, V. 3.50-57, pp. 545-550, and V. 4.10, pp. 560, 561]. N. B.: Rad6's definition of $A^{*}(f, P)$ differs slightly from that above, since (1.4) is more stringent than his requirement of uniform convergence on $P$ of $f_{n}(x, y)$ to $f(x, y)$ : however, it may easily be verified that this difference does not disturb the appeals to his results on $A^{*}(f, P)$ made below (at (1.7), (1.9), and the end of $\$ 5$ ). Figures in square brackets refer to the references at the end of the paper.

(3) For the theorem below I have given a proof in a forthcoming paper [5]: a somewhat less sharp result, not requiring the inscribed polyhedra to be of the form $z=f_{n}(x, y)$, was obtained independently by A. Mambriani [3], whose main result covers a wider class of surfaces. In both papers the proofs are independent of previous work on Geöcze's problem. 
Theorem. If $f(x, y)$ is one-valued and continuous on the unit square $Q_{0}: 0 \leqq x \leqq 1,0 \leqq y \leqq 1$, then (cf. (1.5) above) the Lebesgue area

$$
A\left(f, Q_{0}\right)=A^{*}\left(f, Q_{0}\right) \text {. }
$$

Without loss of generality we may suppose (cf. [8, V.3.22, p. 525, and V.3.46, p. 543]) that $f(x, y)$ satisfies (1.2) and that

$$
A\left(f, Q_{0}\right)<\infty \text {. }
$$

Lемма 1. Let $f(x, y)$ satisfy (1.2) and $R$ be an oriented (closed) rectangle in the $(x, y)$-plane. Let $2^{-1} h k \Phi(x, y ; h, k ; f)$ stand for the area of the triangle whose vertices are $(x, y, f(x, y)),(x+h, y, f(x+h, y)),(x, y+k, f(x, y+k))$, and let

$$
I(h, k ; R ; f)=\iint_{R} \Phi(x, y ; h, k ; f) d x d y .
$$

Then (cf. (1.5)), if $q$ is any given nonzero constant,

$$
A^{*}(f, R) \leqq I^{*}(R, f, q)=\lim \sup I(h, k ; R ; f) \quad(h, k \rightarrow 0, h / k=q) .
$$

This differs from Radó's inequality [8, V. 3.53, (7), p. 549] by the restriction $h / k=q$. If $R$ is the rectangle $[a, b ; c, d]$, Radó's proof actually covers the case in which $q(d-c) /(b-a)$ is positive and rational $\left({ }^{4}\right)$. The modifications required for the remaining cases are obvious and so need not be stated here.

2. An upper bound for $I^{*}(R, f, q)-A(f, R)$ in terms of a Tonelli integral.

LeMma 2. Let $f(x, y)$ satisfy (1.2) and (1.7), $R$ be a closed rectangle in the $(x, y)$-plane oriented relative to the axes of $x$ and $y, O \xi, O \eta$ be any axes of rectangular coordinates in this plane, and $m_{\eta}$ be the slope of On relative to the $x$-axis. Let $V_{\xi}(R, \eta, f)$ denote the total variation of $f(x, y)$ on $R$ for fixed $\eta$ and varying $\xi$ (or 0 if $\eta$ does not occur on $R$ ), and let

$$
T_{\xi}(R, f)=\int_{-\infty}^{\infty} V_{\xi}(R, \eta, f) d \eta .
$$

Then, taking $q=m_{\eta}$ when $0<\left|m_{\eta}\right|<\infty$, and $q=1$ otherwise, we have (cf. (1.9))

$$
I^{*}(R, f, q)-A(f, R) \leqq 2 T_{\xi}(R, f) .
$$

Firstly, let $\left|m_{\eta}\right|=\infty$, and, for definiteness, suppose $\xi=x, \eta=y$. Putting $\Delta_{x} f(x, y)=f(x+h, y)-f(x, y), \Delta_{y} f(x, y)=f(x, y+k)-f(x, y)$, we get (cf. 1.8)

$$
\begin{aligned}
\Phi(x, y ; h, k ; f) & =\left[\left(\Delta_{x} f(x, y) / h\right)^{2}+\left(\Delta_{y} f(x, y) / k\right)^{2}+1\right]^{1 / 2} \\
& \leqq\left|\Delta_{x} f(x, y) / h\right|+\left[\left(\Delta_{y} f(x, y) / k\right)^{2}+1\right]^{1 / 2}
\end{aligned}
$$

(4) N.B.: the use of an enclosing rectangle $R^{\delta}$ in Rado's proof, and the consequent appeal in its final step to the elaborate analysis in [8, V. 3.25-36, pp. 527-536], can be avoided if the terms with either $i=m-1$ or $j=n-1$ are omitted from the summation in [8, V. 3.50, (1), p. 545], and the requisite adjustments made in the ensuing argument. 
by elementary calculations. Suppose that $R$ is the rectangle $[a, b ; c, d]$ : then, from the known fact( $\left(^{5}\right)$ that as $h \rightarrow 0$ the integral from $a$ to $b$ of $\left|\Delta_{x} f(x, y) / h\right|$ tends to $V_{x}(R, y, f)$, it can be deduced( $\left.{ }^{5}\right)$ that

$$
\lim _{h \rightarrow 0} \iint_{R}\left|\Delta_{x} f(x, y) / h\right| d x d y=\int_{c}^{d} V_{x}(R, y, f) d y=T_{x}(R, f) .
$$

Further, from the known fact (cf. [9, chap. V, (8.10), p. 184]) that as $k \rightarrow 0$ the integral from $c$ to $d$ of $\left[\left(\Delta_{y} f(x, y) / k\right)^{2}+1\right]^{1 / 2}$ tends to the length $L_{y}(R, x, f)$ of the curve $z=f(x, y)(x=$ constant, $c \leqq y \leqq d)$ it can be deduced similarly that

$$
\lim _{k \rightarrow 0} \iint_{R}\left[\left(\Delta_{y} f(x, y) / k\right)^{2}+1\right]^{1 / 2} d x d y=\int_{a}^{b} L_{y}(R, x, f) d x=S_{y}(R, f),
$$

say. From (2.3), on integrating $\Phi(x, y ; h, k ; f)$ over $R$ and taking upper limits as $h, k \rightarrow 0(h / k=q=1)$, we obtain (cf. (1.8), (1.9), (2.4), (2.5)) the inequality $I^{*}(R, f, 1) \leqq T_{x}(R, f)+S_{y}(R, f)$. But it was proved by Tonelli $[10, \S 1, \mathrm{p}$. $635]$, that $S_{y}(R, f) \leqq A(f, R)$ : thus

$$
I^{*}(R, f, 1)-A(f, R) \leqq T_{x}(R, f) .
$$

This implies (2.2) in the case where $m_{\eta}$ is infinite; the case $m_{\eta}=0$ is treated similarly, with $x$ and $y$ interchanged. Lastly, suppose $0<\left|m_{\eta}\right|<\infty$ and so $h / k=q=m_{\eta}$. For brevity, we shall put $\alpha=h^{2} /\left(h^{2}+k^{2}\right)^{1 / 2}, \beta=k^{2} /\left(h^{2}+k^{2}\right)^{1 / 2}$, $\gamma=(\alpha \beta)^{1 / 2}$; then for the points whose old coordinates are $(x, y),(x+h, y)$, $(x, y+k)$, and $\left(x+h k^{2} /\left(h^{2}+k^{2}\right), y+h^{2} k /\left(h^{2}+k^{2}\right)\right)$ we find easily the new coordinates $(\xi, \eta),(\xi+\alpha, \eta+\gamma),(\xi-\beta, \eta+\gamma)$, and $(\xi, \eta+\gamma)$ respectively. We write $\phi(\xi, \eta)$, and so on, for $f(x, y)$, and so on, and make the following abbreviations:

$$
\begin{aligned}
\phi(\xi+\alpha, \eta+\gamma)-\phi(\xi, \eta+\gamma) & =\lambda \alpha, \\
\phi(\xi-\beta, \eta+\gamma)-\phi(\xi, \eta+\gamma) & =\mu \beta, \\
\phi(\xi, \eta)-\phi(\xi, \eta+\gamma) & =\nu \gamma .
\end{aligned}
$$

Then, for example, $\Delta_{x} f(x, y)=\lambda \alpha-\nu \gamma$, and thus, from the first line of (2.3),

$$
\begin{aligned}
\Phi(x, y ; h, k ; f) & =\left[(\lambda \alpha-\nu \gamma)^{2}\left(\alpha^{2}+\gamma^{2}\right)^{-1}+(\mu \beta-\nu \gamma)^{2}\left(\beta^{2}+\gamma^{2}\right)^{-1}+1\right]^{1 / 2} \\
& \leqq\left\{\frac{\lambda^{2} \alpha^{2}}{\alpha^{2}+\gamma^{2}}+\frac{\mu^{2} \beta^{2}}{\beta^{2}+\gamma^{2}}\right\}^{1 / 2}+\left\{\frac{\nu^{2} \gamma^{2}}{\alpha^{2}+\gamma^{2}}+\frac{\nu^{2} \gamma^{2}}{\beta^{2}+\gamma^{2}}+1\right\}^{1 / 2},
\end{aligned}
$$

by the triangle inequality. Since $\gamma^{2}=\alpha \beta$ the last expression reduces to

$$
\left(\lambda^{2} \alpha+\mu^{2} \beta\right)^{1 / 2}(\alpha+\beta)^{-1 / 2}+\left(\nu^{2}+1\right)^{1 / 2} \leqq|\lambda|+|\mu|+\left(\nu^{2}+1\right)^{1 / 2} .
$$

Hence, recalling the definition of $I^{*}(R, f, q)$ in Lemma 1, we have

(5) Cf. [8, III. 2.42, p. 207, and V. 3.26, p. 528]; see also [4, (12.2), p. 309]. Note that $T_{x}(R, f)$ is one of Tonelli's integrals, cf. [8, III. 2.49-51, pp. 210-212]. 


$$
\begin{aligned}
I^{*}(R, f, q) \leqq \lim \sup \left\{\iint_{R}|\lambda| d \xi d \eta\right. & +\iint_{R}|\mu| d \xi d \eta \\
& \left.+\iint_{R}\left(\nu^{2}+1\right)^{1 / 2} d \xi d \eta\right\},
\end{aligned}
$$

as $\alpha \rightarrow 0$ with $\beta=\alpha / q^{2}$ and $\gamma=(\alpha \beta)^{1 / 2}$. But, in virtue of (2.7) the last integral in (2.8) has essentially the same form $\left(^{6}\right)$ as the first in (2.5), and the other two integrals in (2.8) can be reduced to essentially the same form $\left(^{6}\right)$ as the first in (2.4) by change of origin to $\xi=0, \eta=-\gamma$. The deduction of the required inequality (2.2) can thus be completed in the same way as that of (2.6) above.

3. Use of W. H. Young's vector-areas to orient the auxiliary axes $O \xi$, $O \eta$. Let $R$ be any rectangle (oriented or not) in the plane $z=0$. Then (cf. (1.1)), for the vector-area (cf. [12]) of $S(f, R)$-or rather, of its boundarycurve $C(R)$-we may write

$$
Y(R, f)=\frac{1}{2} \int_{C(R)} \boldsymbol{r} \times d \boldsymbol{r}, \quad Y(R, f)=|\boldsymbol{Y}(R, f)| .
$$

If we write $c(R)$ for the boundary of $R$, described positively, then for the components of $Y$ we have $Y_{z}(R, f)=Y_{z}(R)=|R|$, that is, the area of $R$,

$$
Y_{x}(R, f)=-\int_{c(R)} z d y=-\int d y \int d_{x} f(x, y) \quad[(x, y) \in R],
$$

and similarly for $Y_{y}(R, f)$. Since the inner integral does not exceed $V_{x}(R, y, f)$ in absolute value we have (cf. (2.1) and (2.4))

$$
\left|Y_{x}(R, f)\right| \leqq T_{x}(R, f), \quad\left|Y_{y}(R, f)\right| \leqq T_{y}(R, f) .
$$

We put $T_{z}(R)=|R|=Y_{z}(R)$ and (suppressing arguments) $T=\left(T_{x}^{2}+T_{y}^{2}\right.$ $\left.+T_{z}^{2}\right)^{1 / 2}$. Using (3.3) and the known fact $\left(^{7}\right)$ that $T$ does not exceed the area $A$, we get

$$
T_{x}^{2}=T^{2}-T_{y}^{2}-T_{z}^{2} \leqq T^{2}-Y_{y}^{2}-Y_{z}^{2}=T^{2}-Y^{2}+Y_{x}^{2} \leqq A^{2}-Y^{2}+Y_{x}^{2} .
$$

Similarly if we work with auxiliary coordinate axes $O \xi, O \eta$, we have

$$
\left[T_{\xi}(R, f)\right]^{2} \leqq[A(f, R)]^{2}-[Y(R, f)]^{2}+\left[Y_{\xi}(R, f)\right]^{2} .
$$

${ }^{6}$ ) That $R$ is not oriented relative to the axes of $\xi$ and $\eta$ causes no trouble, since the theorems needed in the subsequent deduction are obtainable by trivial adaptations from those for oriented rectangles: cf. $[4,(12.2)$, p. 309]. The change of origin translates $R$ through a distance $\gamma$, but it can easily be verified that this makes no difference in the limit, when $\gamma \rightarrow 0$, since $T_{\xi}(R, f)$ is a continuous function of $R$ (cf. [8, III. 2.51, pp. 210-212]). $427,428]$.

$(7)$ Cf. $[10, \$ \$ 5$ and 7 , pp. 447,448$]$; for non-oriented $R$ cf. [1, Lemmas 5.4 and 5.5, pp. 
Now from (3.1), $Y(R, f)$ is independent of the choice of coordinate axes; hence (3.4) yields, in particular, the following lemma.

Lemma 3. Let $f(x, y)$ and $R$ be as in Lemma 2, and let us choose axes $O \xi$, $O \eta$ of rectangular coordinates in the $(x, y)$-plane such that $O \xi$ is perpendicular to $Y(R, f)(c f .(3.1))$. Then (cf. (2.1) and $\$ 1$, below (1.5))

$$
\left[T_{\xi}(R, f)\right]^{2} \leqq[A(f, R)]^{2}-[Y(R, f)]^{2} \leqq 2 A(f, R) D(f, R),
$$

where $D(f, R)$ stands for $A(f, R)-Y(R, f)$.

Corollary to Lemmas 1, 2, AND 3. With $f(x, y)$ and $R$ as in Lemma 2 we have

$$
A^{*}(f, R)-A(f, R) \leqq[8 A(f, R) D(f, R)]^{1 / 2} .
$$

4. In general, $A^{*}(f, R)$ increases (broadly) by subdivision.

Lemma 4. Let $f(x, y)$ and $R$ be as in Lemma 1 , and let $R$ be divided into two oriented rectangles $R_{1}$ and $R_{2}$ by a line segment l. Then (cf. (1.5))

$$
A^{*}(f, R) \leqq A^{*}\left(f, R_{1}\right)+A^{*}\left(f, R_{2}\right),
$$

provided that the vertical plane section through $l$ of $S(f, R)$ is rectifiable.

Suppose, for definiteness, that $R$ is the rectangle $[a, b ; c, d]$, that $l$ is the line $x=x^{\prime}, c \leqq y \leqq d$, where $a<x^{\prime}<b$, and that $x \leqq x^{\prime}$ in $R_{1}, x \geqq x^{\prime}$ in $R_{2}$. From (1.5) it follows that there are sequences $\left\{f_{n}^{i}(x, y)\right\}, i=1,2$, such that $f_{n}^{l}(x, y)$ satisfies on $R_{i}$ the conditions (1.3) and (1.4) and also

$$
\begin{aligned}
E\left(f_{n}^{i}, R_{i}\right) & \rightarrow A^{*}\left(f, R_{i}\right) \text { as } n \rightarrow \infty \quad(i=1,2) ; \\
\tau_{n}=\max \operatorname{diam} \Delta \rightarrow 0 \text { as } n \rightarrow \infty, & \left(\Delta \in \mathcal{T}_{n}^{i}\left(R_{i}\right), i=1,2\right),
\end{aligned}
$$

where $\mathcal{T}_{n}^{i}\left(R_{i}\right)$ is the triangulation of $R_{i}$ for $f_{n}^{i}(x, y)$ (cf. (1.3) and (1.4)).

Now suppose that $\Delta$ is any triangle of $\mathcal{T}_{n}^{1}\left(R_{1}\right)$ that has two vertices $P_{0}$ and $P_{0}^{\prime}$ on $l$ and the third $C_{0}$ thus in $R_{1}-l$. Then we subdivide $\Delta$, when possible, by lines joining $C_{0}$ to each vertex of $\mathcal{T}_{n}^{2}\left(R_{2}\right)$ lying on $l$ strictly between $P_{0}$ and $P_{0}^{\prime}$. Let $\mathcal{\sigma}_{n}\left(R_{1}\right)$ be the triangulation obtained from $\mathcal{\sigma}_{n}^{1}\left(R_{1}\right)$ after all such subdivisions, and let $\mathcal{T}_{n}\left(R_{2}\right)$ be constructed similarly: then we can combine $\mathcal{T}_{n}\left(R_{1}\right)$ and $\mathcal{T}_{n}\left(R_{2}\right)$ into a single triangulation $\mathcal{T}_{n}(R)$ of $R$, since $\mathcal{T}_{n}\left(R_{1}\right)$ and $\mathcal{T}_{n}\left(R_{2}\right)$ have the same vertices on $l$. Clearly $\mathcal{T}_{n}(R)$ satisfies (1.4), and determines a function $f_{n}(x, y)$ on $R$ in accordance with (1.3).

With $\Delta$ as above, let $\mathcal{T}_{n}(\Delta)$ be the triangulation of $\Delta$ under $\mathcal{T}_{n}(R)$, let $A_{0} B_{0} C_{0}$ be a triangle $\delta$ of $\mathcal{T}_{n}(\Delta)$, and let $A B C$ be the corresponding face $S\left(f_{n}, \delta\right)$ (cf. (1.1), (1.3)). Then $E\left(f_{n}, \delta\right)=2^{-1} b c \sin A \leqq 2^{-1} b c$, and so

$$
\begin{aligned}
E\left(f_{n}, \Delta\right)=\sum E\left(f_{n}, \delta\right) \leqq 2^{-1} \sum b c & \leqq 2^{-1} \max b \cdot \sum c \quad\left[\delta \in \mathcal{T}_{n}(\Delta)\right] \\
& \leqq 2^{-1} \max b \cdot L_{y}\left(\Delta, x^{\prime}, f\right),
\end{aligned}
$$


since $\sum c=L_{y}\left(\Delta, x^{\prime}, f_{n}\right)$, where $L_{y}$ is defined as before (2.5). Next, if $(x, y)$ and $(u, v)$ are two points varying on $R$, we write $\omega(\lambda)$ for $\max \mid f(x, y)$ $-f(u, v) \mid$ when $(x-u)^{2}+(y-v)^{2} \leqq \lambda^{2}$. But $\max A_{0} C_{0} \leqq \operatorname{diam} \Delta \leqq \tau_{n}$, by (4.3); and so we get successively

$$
\begin{gathered}
\max b=\max A C \leqq \sigma_{n}=\left[\tau_{n}^{2}+\omega\left(\tau_{n}\right)^{2}\right]^{1 / 2}, \\
E\left(f_{n}, \Delta\right)-E\left(f_{n}^{1}, \Delta\right) \leqq 2^{-1} \sigma_{n} L_{y}\left(\Delta, x^{\prime}, f\right)
\end{gathered}
$$

by (4.4), since $E\left(f_{n}^{1}, \Delta\right)>0$. Further, any triangle $\Delta$ of $\mathcal{T}_{n}^{1}\left(R_{1}\right)$ not adjacent to $l$ is not subdivided, and hence it satisfies (4.6) trivially since both sides vanish. Thus, summing (4.6) over all triangles $\Delta$ of $\mathcal{T}_{n}^{1}\left(R_{1}\right)$, we get

$$
\begin{aligned}
E\left(f_{n}, R_{1}\right)-E\left(f_{n}^{1}, R_{1}\right) & =\sum\left\{E\left(f_{n}, \Delta\right)-E\left(f_{n}^{1}, \Delta\right)\right\} \quad\left[\Delta \in \mathcal{T}_{n}^{1}\left(R_{1}\right)\right] \\
& \leqq 2^{-1} \sigma_{n} \sum L_{y}\left(\Delta, x^{\prime}, f\right)=2^{-1} \sigma_{n} L_{y}\left(R, x^{\prime}, f\right) .
\end{aligned}
$$

Similarly for $R_{2}$; and so, since $E\left(f_{n}, R\right)=E\left(f_{n}, R_{1}\right)+E\left(f_{n}, R_{2}\right)$, we have

$$
E\left(f_{n}, R\right)-E\left(f_{n}^{1}, R_{1}\right)-E\left(f_{n}^{2}, R_{2}\right) \leqq \sigma_{n} L_{y}\left(R, x^{\prime}, f\right) .
$$

On taking upper limits as $n \rightarrow \infty$ we obtain from this, using (4.2),

(4.8) $\lim \sup E\left(f_{n}, R\right)-A^{*}\left(f, R_{1}\right)-A^{*}\left(f, R_{2}\right) \leqq \lim \sup \sigma_{n} L_{y}\left(R, x^{\prime}, f\right)=0$;

for, using (4.3) and (4.5), we see that (as $n \rightarrow \infty) \omega\left(\tau_{n}\right) \rightarrow 0$ because $f(x, y)$ is continuous on $R$, and so $\sigma_{n} \rightarrow 0$; moreover, $L_{y}\left(R, x^{\prime}, f\right)$ is finite by hypothesis. But the desired inequality (4.1) follows from (4.8) and (1.5).

5. Proof of the theorem. As noted in $\$ 1$, we may assume (1.2) and (1.7): further, we employ the notations of $\$ 1$ and Lemma 3, but with the argument $f$ suppressed. Then, following Radó $[6, \S 1.6$, p. $362, \S 2.4$, p. 364 , and $\S 3.8$ including footnote 14, p. 370 ] we note that there is a sequence of subdivisions $\left\{\mathcal{D}_{n}\right\}$ of $Q_{0}$ into oriented rectangles such that (i) the division-lines run from side to side of $Q_{0}$ and are the projections of rectifiable vertical sections of $S\left(f, Q_{0}\right)$, (ii) we have

$$
\begin{aligned}
& \sum D(R)=\sum A(R)-\sum Y(R)=A\left(Q_{0}\right)-\sum Y(R) \rightarrow 0 \\
&\left(R \in \mathcal{D}_{n}, n \rightarrow \infty\right),
\end{aligned}
$$

the second equality holding because $A(R)$ is additive (cf. [8, V. 3.18, p. 524]). Now, for a fixed $\mathcal{D}_{n}$, summation of (3.6) and use of Cauchy's inequality yield

$$
\sum A^{*}(R)-\sum A(R) \leqq \sum 1[8 A(R) D(R)]^{1 / 2} \leqq\left[8 \sum A(R) \sum D(R)\right]^{1 / 2} .
$$

But $A^{*}\left(Q_{0}\right)=A^{*}\left(\sum R\right) \leqq \sum A^{*}(R)$ by repeated application of Lemma 4, $\sum A(R)=A\left(Q_{0}\right)<\infty$ by $(1.7)$, and $\sum D(R) \rightarrow 0$ as $n \rightarrow \infty$ by (5.1); hence (5.2) implies that $A^{*}\left(Q_{0}\right)-A\left(Q_{0}\right) \leqq 0$. Since $A^{*}\left(Q_{0}\right) \geqq A\left(Q_{0}\right)$ trivially (cf. [8, V. 3.46, (1), p. 543]) we now have the desired equality (1.6). 


\section{BIBLIOGRAPHY}

1. H. Federer, Surface area. I, Trans. Amer. Math. Soc. vol. 55 (1944) pp. 420-437.

2. H. D. Huskey, Further contributions to the problem of Geöcze, Duke Math. J. vol. 11 (1944) pp. 333-339.

3. A. Mambriani, Sul problema di Geöcze, Annali della Scuola Normale Superiore di Pisa (2) vol. 13 (1944) pp. 1-17 (issued 1948).

4. H. P. Mulholland, On the total variation of a function of two variables, Proc. London Math. Soc. (2) vol. 46 (1940) pp. 290-311, and vol. 50 (1949) pp. 559-560.

5. - Solution of Geöcze's problem for a continuous surface $z=f(x, y)$, to be published in Proc. London Math. Soc.

6. T. Rad6, On a problem of Geöcze, Amer. J. Math. vol. 65 (1943) pp. 361-381. 506.

7. - Some remarks on the problem of Geöcze, Duke Math. J. vol. 11 (1944) pp. 497-

8. - Length and area, Amer. Math. Soc. Colloquium Publications, vol. 30, New York, 1948.

9. S. Saks, Theory of the integral, 2d ed., Warsaw, 1937.

10. L. Tonelli, Sulla quadratura delle superficie, Rendiconti della R. Accademia Nazionale Lincei (6) vol. 3 (1926) pp. 445-450 and 633-638.

11. L. C. Young, $A n$ expression connected with the area of a surface $z=F(x, y)$, Duke Math. J. vol. 11 (1944) pp. 43-57.

12. W. H. Young, On the area of surfaces, Proc. Royal Soc. London ser. A vol. 96 (1920) pp. 71-81.

American University, BEIRUT, LEBANON. 